\title{
Pattern of Antimicrobial Resistance among Bacterial Isolates from Urogenital Clinical Specimens: A Descriptive Study from the Buea Health District, Cameroon
}

\author{
Elvis Tajoache Amin ${ }^{1,2,3} \mathbb{C}^{-}$Charles Njumkeng ${ }^{1,2,3} \cdot$ Belmond T. Kika $^{3,4,5}$ • \\ Akemfua Fualefac ${ }^{6} \cdot$ Patrick Njukeng $^{6}$
}

Published online: 12 April 2018

(C) The Author(s) 2018

\begin{abstract}
Introduction Antimicrobial resistance has become a global concern and is particularly affecting developing countries where infectious diseases and poverty are endemic. The effectiveness of currently available antimicrobials is decreasing as a result of increasing resistant strains among clinical isolates.

Objectives The aim of this study was to determine the resistance pattern of bacterial isolates from different clinical urogenital specimens at different hospitals in the Buea Health District, Cameroon.

Methods A retrospective study was conducted in three hospital laboratories in the Buea Health District,
\end{abstract}

Elvis Tajoache Amin

Charles Njumkeng

njumkengcharles@yahoo.com

Belmond T. Kika

belmondkika@gmail.com

Akemfua Fualefac

a_fualefac@ghsscm.org

Patrick Njukeng

p3njukeng@gmail.com

1 St. Albert The Great Reference Medical Diagnostic Center, Buea, Cameroon

2 Global Research Education and Health Foundation, Buea, Cameroon

3 Ministry of Public Health, Yaounde, Cameroon

4 Atlantic Medical Foundation Hospital, Mutengene, Cameroon

5 Clinical Research Education, Networking and Consultancy, Douala, Cameroon

6 Global Health Systems Solutions, SONARA Road, Limbe, Cameroon
Cameroon, from June to August 2017. All culture and antimicrobial susceptibility test results of patients who presented at each of the laboratories for urine, vaginal swab or urethral swab cultures from January 2012 to December 2016 were included in the study. Data were analysed using SPSS Windows version 20.0. The comparisons between different isolates' resistance to antimicrobials were performed using the chi-square test. The difference in the resistance of urogenital isolates to various antimicrobials within different years was also compared by the chi-square test.

Results A total of 423 bacterial isolates were obtained from clinical urogenital specimens such as: urine 93 (21.9\%), vaginal swab $175(41.4 \%)$ and urethral swab cultures $155(36.6 \%)$. The predominant bacterial isolates were Staphylococcus spp. 320 (75.5\%), Escherichia coli 37 (8.7\%) and Enterococcus spp. 24 (5.7\%). All the isolates showed significantly high resistance rates to amoxicillin/clavulanic acid (67.6\% resistant rate, $p=0.025)$, but most isolates, except those of Staphylococcus, were relatively more susceptible to nitrofurantoin $(82.6 \%$ susceptibility rate, $p=0.045)$. However, Staphylococcus spp. was more susceptible to ceftriaxone $(91.0 \%$ susceptibility rate, $p<0.0001)$ and cefotaxime $(74.4 \%$ susceptibility rate, $p=0.034)$. Generally, most of the isolates showed significantly rising rates of resistance to the majority of the antimicrobials tested from 2012 to 2017.

Conclusion Our findings showed a progressively rising rate of antimicrobial resistance in urogenital bacterial isolates over the last 5 years in the Buea Health District. Thus, uncontrolled and irrational use or prescription of these drugs should be avoided to maintain low resistance of highly susceptible antimicrobials. 


\section{Key Points}

In Cameroon, lack of bacterial testing, circulation of fake drugs, and irrational use of un-prescribed antimicrobials has led to a progressive rise in antimicrobial resistance.

Our findings showed a progressively rising rate of antimicrobial resistance in the uropathogens isolated, with highest rates of resistance to amoxicillin/clavulanic acid while most pathogens, except Staphylococcus spp., remain susceptible to nitrofurantoin.

Uncontrolled and irrational use or prescription of antimicrobials should be avoided so as to maintain low resistance of highly susceptible antimicrobials.

\section{Introduction}

Over the past years, antimicrobial resistance (AMR) has become a global concern. It has particularly affected developing countries where infectious diseases are endemic [1]. Infections that are caused by resistant bacteria have often been associated with increased morbidity and mortality compared with those caused by susceptible pathogens [2]. Infections of the urogenital system are among the commonest bacterial infections encountered in daily clinical practice [3]. Urinary tract infections (UTIs) are a major cause of morbidity in both the hospital and community settings, occurring in all age groups, with most of the infections being treated empirically with broadspectrum antimicrobials. However, antimicrobial resistance in bacteria causing UTIs has progressively increased since the introduction of UTI chemotherapy $[4,5]$.

Several studies [6, 7] show geographic variations in bacterial uropathogens and their resistance patterns to antimicrobials. Escherichia coli, Klebsiella, Pseudomonas aeruginosa and Enterococcus species were the most common bacterial pathogens isolated from the urinary tracts of infected patients $[8,9]$. The antimicrobial susceptibility patterns of bacteria isolated from urinary tracts differ for different bacteria and antimicrobials [8, 10, 11], with increased susceptibility to the quinolones and increased resistance to nitrofurantoin, ampicillin and cotrimoxazole.

E. coli has been reported in studies in Nigeria and Turkey as the most resistant microorganism, $[8,12]$ contributing to recurrent infections [13].

In Cameroon and other resource-limited countries, treatment choices for the majority of urinary and other genital tract infections (pelvic inflammatory diseases, urethritis, etc.) are empirically based on the predictable spectrum of aetiological micro-organisms and available data reflecting antimicrobial resistance of previous infections [6]. Lack of bacterial testing, circulation of fake drugs, and irrational use of un-prescribed antimicrobials are the possible reasons for antimicrobial resistance, and are responsible for recurrence of infections as well as complicated UTIs [12, 14, 15]. Recent studies show a growing problem of antimicrobial resistance in Cameroon $[11,16,17]$, thereby necessitating the need for continuous surveillance of antimicrobial susceptibility to uropathogens.

\section{Methods}

\subsection{Study Area and Sites}

This study was conducted in the Buea Health District, Cameroon. The Buea Health District is among the four health districts that make up the Fako Division of Cameroon with a total population of 131,325 [18]. It has an average temperature, humidity and rainfall of $23{ }^{\circ} \mathrm{C}, 83.5 \%$ and $58 \mathrm{~mm}$, respectively. The Buea Health District is divided into seven health areas, which include: Molyko, Muea, Buea Town, Bova, Bokwaongo, Tole and Buea Road health areas. The health district has 66 communities and a total of 21 health facilities (both private and state owned). The study was conducted in all eligible laboratories within the health district where culture and sensitivity testing is done.

\subsection{Study Design and Population}

A retrospective study was conducted in three health facilities (St Albert Medical Centre, Mount Mary Hospital and Solidarity Clinic) in the Buea Health District, Cameroon, from June to August 2017. All cultures and antimicrobial susceptibility test results of patients who presented at each of the laboratories for urine, vaginal swab or urethral swab cultures from January 2012 to December 2016 were included in the study. All data were extracted from laboratory records using a structured checklist.

\subsection{Ethical Considerations}

Ethical approval was obtained from the Cameroon Baptist Convention Health System Institutional review board (CBCHB IRB) (Re: IRB2017-23).

Administrative authorization for this study was obtained from the South West Regional Delegation of Public Health. Permission to collect the information from registers was 
obtained from the administration of the various hospitals involved. Considering that the study does not directly involve human subjects a consent waiver was applied. All data collected were treated with strict confidentiality through coding (anonymous) of the cases. Within each selected laboratory, the data were extracted by the laboratory staff to avoid breaching the patient's confidentiality since the registers have patient names.

\subsection{Data Collection}

After obtaining ethical clearance and administrative authorization, a modified WHO Antimicrobial Resistance Surveillance Questionnaire was used to assess the laboratory's capacity to perform culture and sensitivity testing [19]. The data quality was assessed (for availability, consistency and completeness) as proposed by the DAMA UK Working Group on 'Data Quality Dimensions' [20]. Given the doubtful quality of data from our health system, lack of studies in this area and the uncertainty of compliance with data management practices, we anticipated that an overall data quality of $60 \%$ was reasonable enough to be included in the study. Therefore, $60 \%$ was set as the minimum score for any facility to be included in the study. The data on culture results (isolates and sensitivity results) and demographics (age and sex) were collected with the use of a checklist drawn from the laboratory registers.

\subsection{Sample Collection and Processing}

The samples collected in this study were urine, urethral swabs and vaginal swabs. For urine samples, about $50 \mathrm{ml}$ of midstream urine specimen was collected in a sterile Jeanne specimen container. The semi-quantitative technique to determine significant bacteriuria was employed by using $0.01 \mathrm{ml}$ calibrated wire loop to inoculate the urine on 5\% blood agar, eosin-methylene blue (EMB) agar and CLED agar (cystine lactose-electrolyte-deficient). A urine specimen was considered positive if a single organism was isolated at a concentration greater than $10^{5} \mathrm{CFU} / \mathrm{ml}$ and with a corresponding microscopy finding of greater than 10 leucocytes per high-power field.

For the urethral swab, the urethra opening was cleared using a swab moistened with sterile physiological saline. After inoculation of media, the remaining sample was centrifuged at $2000 \mathrm{rpm}$ for $5 \mathrm{~min}$ and the sediment was used for microscopy.

Vaginal swabs were collected with the aid of a speculum. They were moistened with warm sterile water and inserted into the vagina following the guidelines found in the second edition of Cheesbrough [21]. Vaginal and urethral specimens were inoculated on Sabouraud agar, blood agar, chocolate agar, Mannitol salt agar and EMB agar.
Culture plates were incubated at $37{ }^{\circ} \mathrm{C}$ for $18-24 \mathrm{~h}$. Gram's stain and biochemical procedures were then used to identify the bacteria.

\subsection{Antimicrobial Susceptibility Testing}

Antimicrobial susceptibility was determined using the disc diffusion method on Mueller Hinton agar as described in the guidelines of the Clinical and Laboratory Standard Institute [22]. The families of drugs studied were quinolones (norfloxacin, ofloxacin and ciprofloxacin), nitrofuran (nitrofurantoin), cephalosporins (cefuroxime, ceftriaxone and cefotaxime), penicillins (amoxicillin, piperacillin, amoxicillin/clavulanic acid and ampicillin), macrolides (clarithromycin and erythromycin), aminoglycosides (gentamicin, rifampicin and vancomycin) and tetracyclines (tetracycline and doxycycline).

The plates were incubated for $24 \mathrm{~h}$ against Staphylococuss spp. and 16-18 h for all other isolates. The diameters of the zones of complete inhibition (as judged by the unaided eye) were measured, including the diameter of the disk to the nearest whole millimetre. The diameter was compared to the critical values of each antimicrobial disc to qualify the target bacteria as sensitive, resistant or intermediate. Control tests were performed with Staphylococcus aureus ATCC25923 and E. coli ATCC25922 reference strains.

\subsection{Data Analysis}

Data analysis was done with SPSS version 20 (IBM, Chicago, IL, USA). Comparison between different isolates' resistance to an antimicrobial was performed using the chisquare test. The difference in the resistance of urogenital bacteria to various antimicrobials over different years was also compared by the chi-square test, while Pearson's correlation was used to study the trends of antimicrobial resistance over time. The threshold for statistical significance was $p<0.05$.

\section{Results}

A total of 502 records were reviewed within the study period. Out of the 502 records reviewed, 79 had incomplete data and were rejected. The remaining 423 records were included in the study. Of the total 423 records reviewed, 93 $(21.9 \%)$ were urine culture, $175(41.4 \%)$ were vaginal smear culture and $155(36.6 \%)$ were urethral smear culture. The majority of the records reviewed were of female patients $243(57.3 \%)$. The age of the study participants ranged from 3 years to 83 years, with the mean age of 33.5 years $(S D \pm 14.3)$. Most of the participants were 
within the sexually active age groups of 20-39 years (271 patients; $63.9 \%$ ), while the elderly (age $>60$ years) were the least represented $(30 ; 7.1 \%)$. All records reviewed had at least one bacterial isolate. Eight different species of urogenital pathogens were isolated. All staphylococcus species ( $S$. aureus and $S$. saprophyticus) were group together while $E$. coli and the remaining isolates were also grouped together to facilitate data analysis, since the number of some pathogens isolated was too small to be analysed individually. Among the isolates, Staphylococcus spp.-320 (75.5\%), E. coli-37 (8.7\%) and Enterococcus spp.-24 (5.7\%), were the most predominant (Table 1).

All pathogens isolated were classified as either resistant or susceptible to each of the antimicrobials tested. Staphylococcus spp. and most of the other isolates (dominated by $E$. coli) showed significantly high resistance to amoxicillin/clavulanic acid, with resistance rates of 45.3 and $67.6 \%$, respectively. On the other hand, all isolates except Staphyloccocus spp. were relatively more susceptible to nitrofurantoin $(82.6 \%$ susceptibility rate, $p=0.045)$. However, Staphylococcus spp. demonstrated a greater susceptibility to ceftriaxone with a low resistance rate of 9\%. All the isolates also demonstrated a high rate of

Table 1 Distribution of study participants from various laboratories according to sex, age, specimen and isolates

\begin{tabular}{lc}
\hline Variable & $\begin{array}{l}\text { Frequency } N(\%) \\
N=423\end{array}$ \\
\hline Sex & \\
Female & $243(57.3)$ \\
Male & $180(42.7)$ \\
Age group in years & \\
$<20$ & $36(8.5)$ \\
$20-39$ & $271(63.9)$ \\
$40-59$ & $87(20.5)$ \\
$>60$ & $30(7.1)$ \\
Specimen & \\
Urine & $93(21.9)$ \\
Vaginal smear & $175(41.4)$ \\
Urethral smear & $155(36.6)$ \\
Isolate & \\
Staphylococcus aureus & $286(67.6)$ \\
Staphylococcus saprophyticcus & $34(8.0)$ \\
Streptococcus & $13(3.1)$ \\
Enterococcus spp. & $24(5.7)$ \\
Neisseria gonorrhoeae & $12(2.8)$ \\
Escherichia coli & $37(8.7)$ \\
Klebsiella spp. & $5(1.2)$ \\
Pseudomonas aeruginosa & $1(0.2)$ \\
Proteus spp. & $11(2.6)$ \\
\hline
\end{tabular}

resistance to tetracycline, though this was not statistically significant (Fig. 1).

A total of 18 antimicrobials were analysed over a period of 5 years. It was observed that the isolated pathogens had average resistance of over $50 \%$ to six antimicrobials (tetracycline, erythromycin, amoxicillin/clavulanic acid, clarithromycin, ampicillin and amoxicillin) throughout the study period. The highest resistance rates were seen with ampicillin and tetracycline, with average resistant rates of 64.9 and $64.0 \%$, respectively. On the other hand, four drugs (cefotaxime, ceftriaxone, ciprofloxacin and gentamicin) showed resistance rates below 20\%, and all the isolated uropathogens were most susceptible to gentamicin (as shown in Table 2). All the isolated pathogens demonstrated a fivefold increase in resistance to cefotaxime from 2012 and 2016. Similarly, there was a progressive increase in resistance to amoxicillin from $20 \%$ in 2012 to over $80 \%$ in 2016. No significant increase in resistance to ciprofloxacin, ceftriaxone and gentamicin between 2012 and 2015 was noted. However, there was a dramatic rise in resistance rates to ciprofloxacin from $6.1 \%$ in 2015 to $38.2 \%$ in 2016 .

All isolates found in this study showed a positive correlation of their resistance rate to antimicrobials over the years except for tetracycline. However, only gentamicin showed a strong correlation $(r=0.900, \quad p=0.037)$ (Table 3).

\section{Discussion}

Infections of the urogenital system are among the most common bacterial infections encountered in daily clinical practice. Uropathogens and their antibiotic susceptibility pattern have continued to change [23]. This study determined the resistance pattern of bacterial isolates from different clinical urogenital specimens, at different hospitals in the Buea Health District, Cameroon. The most predominantly isolated bacteria were Staphylococcus spp., E. coli and Enterococcus spp. Similar findings have been reported in two studies in Ethiopia [24, 25]. The high rate of Staphylococcus spp. isolated was mainly because the different subtypes of staphylococci were grouped together since most of them were not well represented. However, $S$. aureus was the most predominant subtype and can be attributed to the patients' normal endogenous flora [16]. The second highest isolate in our study was E. coli. It has been the most frequently reported isolate causing urinary tract infections in similar studies $[6,11,12]$. The lower number of isolated $E$. coli from our study can be explained by the fact that the data we obtained from urine cultures accounted for only $36.6 \%$; in addition, bacterial isolates vary with different clinical specimens, study designs, geographic locations and study populations. 
Fig. 1 Comparison of the resistance of Staphylococcus isolates and all other isolates to the various antimicrobials from selected laboratories in the Buea Health District, Cameroon, from 2012 to 2017

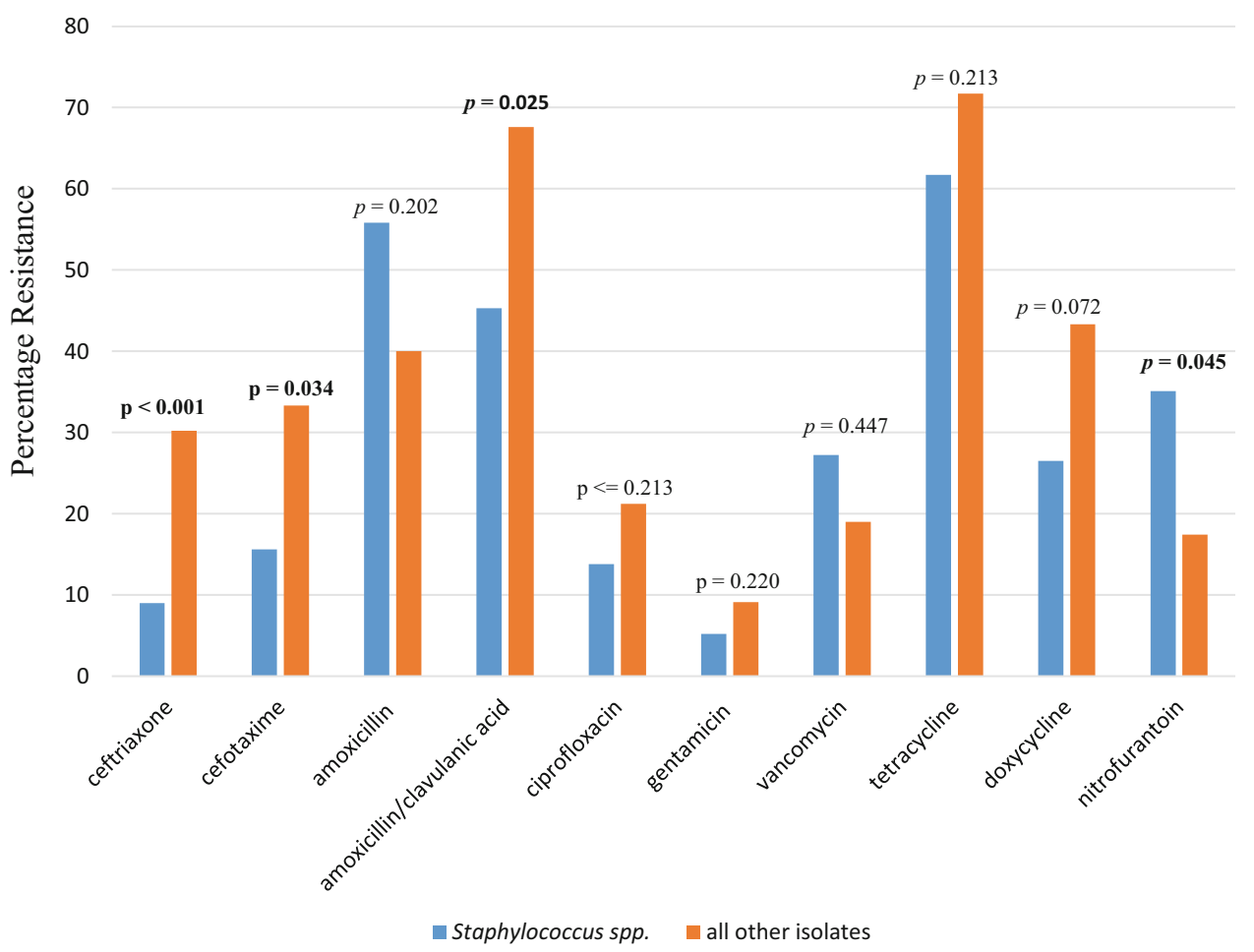

With regard to the pattern of antimicrobial resistance to the different isolates, Staphylococcus spp. showed a significantly high resistance rate to nitrofurantoin compared to all other isolates. On the other hand, all other isolates were more resistant to ceftriaxone, cefotaxime and amoxicillin/clavulanic acid. The high resistance rate of Staphylococcus spp. to these drugs limits their use as empirical treatment of urinary tract as well as other genital tract infections. A high rate of resistance to amoxicillin has also been reported in Nigeria [26]. Also, Staphylococcus spp. are normal body flora and frequently found in the environment with consistent human infection, thus the resistance profile of Staphylococcus spp. to commonly available antimicrobials such as amoxicillin may not be unexpected in environments with frequent self-medication and irrational use of un-prescribed antimicrobials. Staphylococcus spp. on the other hand were relatively more susceptible to ceftriaxone and cefotaxime, with relatively low resistance rates. This can be explained further by the fact that these particular drugs are not readily available in most pharmacies and drug stores in the form of tablets but rather as injections. Thus, they are not common at most roadside drug vendors and some pharmacies for easy consumption without prescription, thereby keeping the rate at which the drugs can be misused to relatively low levels. This finding was also in accordance with results by Alo et al. in Nigeria [21].

The other isolates found in this study (E. coli, Enterococcus spp., Neisseria gonorrhoeae, Klebsiella,
Pseudomonas aeruginosa and Proteus spp.) together showed a significant susceptibility to nitrofurantoin. The high resistance rate of the other isolates, particularly E. coli, which was most predominant, has also been reported by Yaounde [7]. This increasing resistance rate could be attributed to the rapidly growing population as well as to progressive urbanization of the Buea Health District, which now has greater access to antimicrobials, hence a greater consumption rate leading to an increase in resistance [11]. It was also noted that all the urogenital pathogens isolated had very high resistance rates to tetracycline, ampicillin and erythromycin, but these rates were not statistically significant. This was in line with findings by Alo et al. [21].

Over the past years, studies have shown the emergence of widespread resistance to commonly available first-line antimicrobial agents (cephalosporins and fluoroquinolones) in sub-Saharan Africa [27]. Likewise, our findings indicate that there is also a gradual increase in resistance of bacterial species to these classes of drugs. This was illustrated by the fivefold increase in resistance to cefotaxime. On the contrary, no significant increase in resistance to ciprofloxacin, ceftriaxone and gentamicin was observed between 2012 and 2015, though there was a sudden rise in ciprofloxacin resistance from $6.1 \%$ in 2015 to $38.2 \%$ in 2016 . This can be accounted for by the fact that microbial resistance to drugs built up over the years. The resistant pattern observed in Senegal, Nigeria and Malawi indicated a statistically significant rise of resistance to ciprofloxacin, 


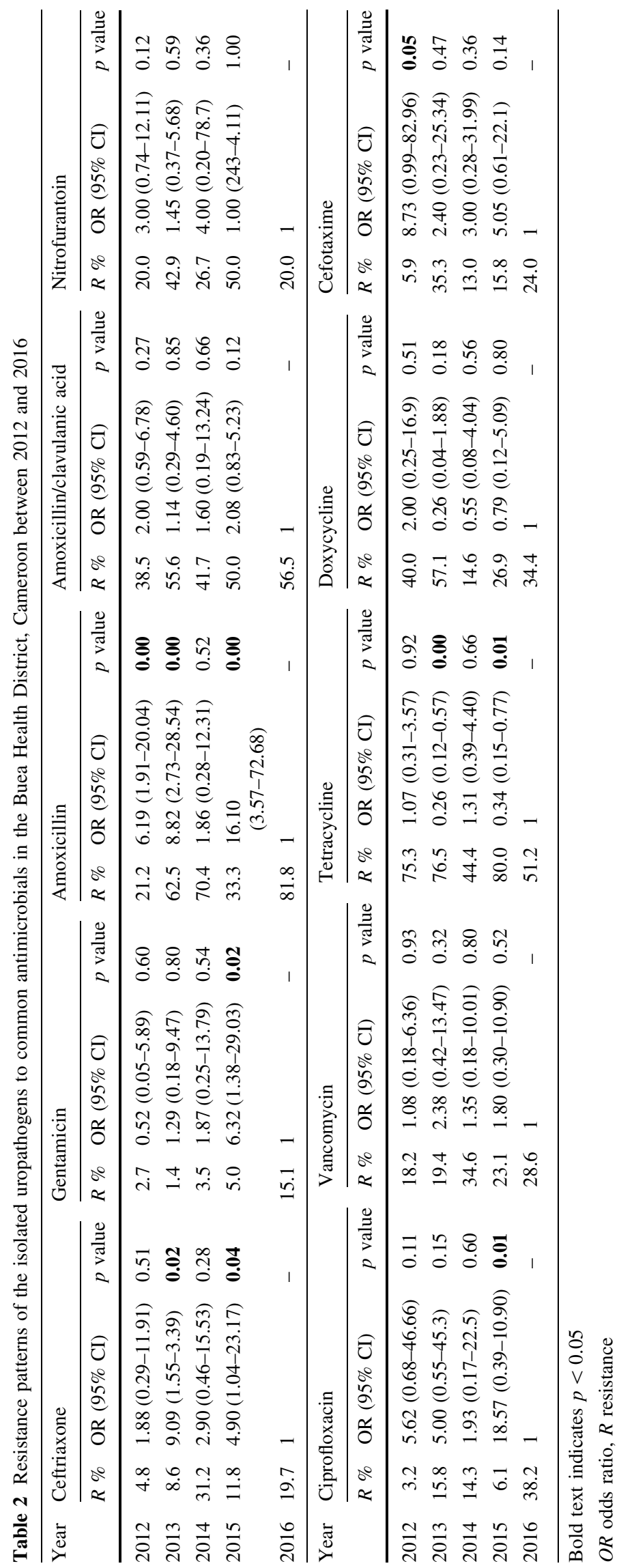


Table 3 Resistance trends of the isolated uropathogens to common antimicrobials in the Buea Health District, Cameroon, between 2012 and 2017

\begin{tabular}{lcc}
\hline Drug & Correction coefficient & $p$ value \\
\hline Ceftriaxone & 0.658 & 0.198 \\
Gentamicin & $\mathbf{0 . 9 0 0}$ & $\mathbf{0 . 0 3 7}$ \\
Amoxicillin & 0.687 & 0.178 \\
Amoxicillin/clavulanic acid & 0.700 & 0.188 \\
Nitrofurantoin & 0.103 & 0.870 \\
Ciprofloxacin & 0.600 & 0.285 \\
Vancomycin & 0.678 & 0.169 \\
Tetracycline & -0.100 & 0.873 \\
Cefotaxime & 0.400 & 0.505 \\
\hline
\end{tabular}

Bold text indicates $p<0.05$

ceftriaxone and gentamicin $[5,28,29]$. This was contrary to our findings and can be explained by the fact that Staphylococcus spp. were the predominant isolates in our study instead of $E$. coli, which was predominant in most of the other studies. Furthermore, a progressive increase in resistance to antimicrobials over the years in this study area can be due to urbanization, since it leads to increases in population, increases in the availability of roadside drug vendors and pharmacies, and poor regulatory mechanisms to prevent the irrational sale and consumption of un-prescribed antimicrobials.

Over the last 5 years (2012-2017), the resistance rate of the bacterial isolates to amoxicillin/clavulanic acid, nitrofurantoin, tetracycline, doxycycline and vancomycin has been progressively rising. This was similar to the resistance trend exhibited by $S$. aureus in a study carried out in Uganda [30]. Due to the retrospective nature of this study, we could not specifically determine the factors that were responsible for this increase in antimicrobial resistance over these years. Thus, we could not adequately explain the fairly high or relatively affected resistance rate to some of the antimicrobials. The small number of records reviewed in the study was also a limitation in adequately describing the resistance pattern to available different antimicrobials of each pathogen isolated. Therefore, we recommend that a prospective study should be carried out to better understand the pattern and associated factors for this progressive increase in antimicrobial resistance.

\section{Conclusion}

This study revealed that Staphylococcus spp. followed by $E$. coli were the most predominant bacterial pathogens in common clinical urogenital specimens. Most pathogens
(Staphylococcus spp., E. coli and Enterococcus spp.) isolated showed significantly high resistance rates to amoxicillin/clavulanic acid, while most of the isolates found in the study, except Staphylococcus spp., remain susceptible to nitrofurantoin. However, Staphylococcus spp. were relatively more susceptible to ceftriaxone and cefotaxime. All pathogens isolated demonstrated a progressive rise in antimicrobial resistance from 2012 to 2016. Thus, the irrational use or prescription of these drugs should be avoided to keep the resistance to highly susceptible antimicrobials low.

Acknowledgements We thank the staff in the file rooms and the data collectors for assisting with the data collection phase of this study. We acknowledge the Directors of St. Albert Clinic, Mount Mary and Solidarity Hospitals who assisted in providing the essential documents required for obtaining ethical clearance.

\section{Compliance with Ethical Standards}

Conflict of interest ETA, NC, BTK, AF and GT have no conflicts of interest.

Funding No external funding was received for this study.

Open Access This article is distributed under the terms of the Creative Commons Attribution-NonCommercial 4.0 International License (http://creativecommons.org/licenses/by-nc/4.0/), which permits any noncommercial use, distribution, and reproduction in any medium, provided you give appropriate credit to the original author(s) and the source, provide a link to the Creative Commons license, and indicate if changes were made.

\section{References}

1. Okeke IN, Laxminarayan R, Bhutta ZA, Duse AG, Jenkins P, O'Brien TF, et al. Antimicrobial resistance in developing countries. Part I: recent trends and current status. Lancet Infect Dis. 2005;5:481-93.

2. Helms M, Vastrup P, Gerner-Smidt P, Mølbak K. Excess mortality associated with antimicrobial drug-resistant Salmonella typhimurium. Emerg Infect Dis. 2002;8:490-4.

3. Moges F, Mengistu G, Genetu A. Multiple drug resistance in urinary pathogens at Gondar College of Medical Sciences Hospital. Ethiopia. East Afr Med J. 2002;79(8):415-9.

4. Orret FA, Davis GK. A comparison of the antimicrobial susceptibility profile of urinary pathogens for the years 1999 and 2003. West Indian Med J. 2006;55:95-9.

5. Omoregie, et al. Observed changes in the prevalence of uropathogens in Benin City, Nigeria. NZ J Med Lab Sci. 2008;62:29-33.

6. Gupta K. Emerging antibiotic resistance in urinary tract pathogens. Infect Dis Clin N Am. 2003;17:321-3.

7. Akoachere J-FTK, Yvonne S, Akum NH, Seraphine EN. Etiologic profile and antimicrobial susceptibility of community-acquired urinary tract infection in two Cameroonian towns. BMC Res Notes. 2012;5:219.

8. Kolawole AS, Kolawole OM, Kandaki-Olukemi YT, Babatunde SK, Durowade KA, Kolawole CF. Prevalence of urinary tract infections (UTI) among patients attending Dalhatu Araf 
Specialist Hospital, Lafia, Nasarawa State, Nigeria. Int J Med Sci. 2009;1:163-7.

9. Khorvash F, Mostafavizadeh K, Mobasherizadeh S, Behjati M. A comparison of antibiotic susceptibility patterns of klebsiella associated urinary tract infection in spinal cord injured patients with nosocomial infection. Acta Med Iran. 2009;47:447-50.

10. Dias-Neto JA, Silva LDM, Martins ACP. Prevalence and bacterial susceptibility of hospital acquired urinary tract infection. Acta Cir Bras. 2003;18(Suppl 5):36-8. https://doi.org/10.1590/ s0102-86502003001200013.

11. Rolf NN, Hortense KG, Sinata K-S. Bacterial, etiology and antibiotic resistance profile of community-acquired urinary tract infections in a Cameroonian City. Int J Microbiol. 2016. https:// doi.org/10.1155/2016/3240268.

12. Ypak C, Altunsoy A, Düzgün N. Empiric antibiotic therapy in acute uncomplicated urinary tract infections and fluoroquinolone resistance: a prospective observational study. Ann Clin Microbiol Antimicrob. 2009;8:27. https://doi.org/10.1186/1476-0711-8-27. https://www.ncbi.nlm.nih.gov/pmc/articles/PMC2770515/. https://www.ncbi.nlm.nih.gov/pubmed/19852849.

13. Mahesh E, Ramesh D, Indumathi VA, Punith K, Kirthi R, Anupama HA. Complicated urinary tract infection in a tertiary care center in South India. AlAmeen J Med Sci. 2010;3(12):120-7.

14. Craig JC, Simpson JM, Williams GJ, Lowe A, Reynolds GJ, McTaggart SJ, Hodson EM, Carapetis JR, Cranswick NE, Smith G, Irwig LM, Caldwell PH, Hamilton S, Roy LP. Antibiotic prophylaxis and recurrent urinary tract infection in children. N Engl J Med. 2009;361:1748-1759. https://doi.org/10.1056/ nejmoa0902295. https://www.ncbi.nlm.nih.gov/pubmed/ 19864673.

15. Nwanze PI, Nwaru LM, Oranusi S, Dimkpa U, Okwu MU, Babatunde BB, Anake TA, Jatto W, Asagwara CE. Urinary tract infection in Okada village: prevalence and antimicrobial susceptibility pattern. Sci Res Essays. 2007;2:112-6.

16. Nkwelang G, Akoachere J-FTK, Kamga LH, Nfoncham ED, Ndip RN. Staphylococcus aureus isolates from clinical and environmental samples in a semi-rural area of Cameroon: phenotypic characterization of isolates. Afr $\mathrm{J}$ Microbiol Res. 2009;3(11):731-6.

17. Ndip RN, Dilonga HM, Ndip LM, Akoachere JFK, Nkuo Akenji T. Pseudomonas aeruginosa isolates recovered from clinical and environmental samples in Buea, Cameroon: current status on biotyping and antibiogram. Trop Med Int Health. 2005;10(1):74-81.

18. Buea (Arrondissement, Cameroon)—population statistics, charts, map. https://www.citypopulation.de/php/cameroon-admin. php?adm2id=100101. Accessed 28 Dec 2017.

19. Antimicrobial resistance surveillance. Questionnaire for Assessment of National Networks. http://www.who.int/drugresistance/
publications/WHO_CDS_CSR_RMD_2003_1/en/. Accessed 9 Sept 2017.

20. DAMA UK Working Group on "Data Quality Dimensions". The six primary dimensions for data quality assessment: defining data quality dimensions. 2013. https://www.dqglobal.com/wp-content/ uploads/2013/11/DAMA-UK-DQ-Dimensions-White-PaperR37. pdf. Accessed 16 Feb 2016.

21. Cheesbrough M. Medical laboratory manual for tropical countries: anatomy and physiology, clinical chemistry and parasitology, 2nd edn. 2006.

22. Clinical and Laboratory Standards Institute for Disk. Volume 32 Document M2-A11. Performance standards for antimicrobial disk susceptibility tests, 11 th edn. 2012.

23. Goldstein FW. Antibiotic susceptibility of bacterial strains isolated from patients with community-acquired urinary tract infections in France. Multicentre Study Group. Eur J Clin Microbiol Infect Dis. 2000;19(2):112-7.

24. Dagnachew M, Yitayih W, Getachew F, Tesfaye N, Kasaw A, Belete B. Bacterial isolates and their antibiotic susceptibility patterns among patients with pus and/or wound discharge at Gondar university hospital. BMC Res Notes. 2014;7:619.

25. Mulu A, Moges F, Tesema B, Kassu A. Pattern and multiple drug resistance of bacterial pathogens isolated from wound infection at University of Gondar teaching hospital, Northwest Ethiopia. Ethiop Med J. 2006;44:125-31.

26. Alo MN, Saidu AY, Ugah UI, Alhassan M. Prevalence and antbiogram of bacterial isolates causing urinary tract infections at federal teaching hospital Abakaliki I (FETHA I). Br Microbiol Res J. 2015;8(2):403-17.

27. Leopold SJ, van Leth F, Tarekegn H, Schultsz C. Antimicrobial drug resistance among clinically relevant bacterial isolates in subSaharan Africa: a systematic review. J Antimicrob Chemother. 2014;69:2337-53.

28. Sire J-M, Nabeth P, Perrier-Gros-Claude J-D, Bahsoun I, SibyT Macondo EA, et al. Antimicrobial resistance in outpatient Escherichia coli urinary isolates in Dakar, Senegal. J Infect Dev Ctries. 2007;1:263-8.

29. Musicha P, Cornick JE, Bar-Zeev N, French N, Masesa C, Denis B, Kennedy N, Mallewa J, Gordon M, Msefula C, Heyderman RS, Everett DB, Feasey NA. Trends in antimicrobial resistance in bloodstream infection isolates at a large urban hospital in Malawi (1998-2016): a surveillance study. Lancet Infect Dis. 2017. https://doi.org/10.1016/s1473-3099(17)30394-8.

30. Joel B, Yap B, Joseph S, Jacobs I, Deborah N, Nelson S, Damalie N. Trends in antimicrobial resistance of Staphylococcus aureus isolated from clinical samples at Mbarara Regional Referral Hospital in Rural Uganda. $\mathrm{Br}$ Microbiol Res J. 2014;4(10):1084-91. 